\title{
Energy Harvesting in a Nonlinear Cantilever Piezoelastic Beam System Excited by Random Vertical Vibrations
}

\author{
Marek Borowiec* and Grzegorz Litak ${ }^{\dagger}$ \\ Department of Applied Mechanics \\ Lublin University of Technology \\ Nadbystrzycka 36, 20-618 Lublin, Poland \\ *m.borowiec@pollub.pl \\ ${ }^{\dagger}$ g.litak@pollub.pl \\ Michael I. Friswell* and Sondipon Adhikari\$ \\ College of Engineering \\ Swansea University, Singleton Park, Swansea SA2 8PP, UK \\ ${ }^{\dagger}$ m.i.friswell@swansea.ac.uk \\ §s.adhikari@swansea.ac.uk \\ Received 3 March 2014 \\ Accepted 30 April 2014 \\ Published 27 August 2014
}

\begin{abstract}
The vertical elastic beam with vertical ambient excitation is proposed as an energy harvester. The beam has a tip mass and piezoelectric patches which transduce the bending strains induced by the stochastic force caused by vertical kinematic forcing into electrical charge. We focus on the region with a fairly large amplitude of voltage output where the beam overcomes the potential barrier. Increasing the noise level allows the transition from single well oscillations to inter-well stochastic jumps with more power generation.
\end{abstract}

Keywords: Mechanical vibration; wideband; energy harvesting; nonlinear; piezoelectric transducer.

\section{Introduction}

Recent activities on self-powered devices, such as sensors and small actuators working in small networks, has stimulated new research ideas on ambient energy harvesting. ${ }^{1-4}$ In competition with photo-voltaic and thermal gradient approaches, frequency broadband nonlinear vibration solutions have been developed. ${ }^{6,7}$ The effectiveness of these devices, based on density of harvested energy, and their availability are continuously growing. ${ }^{5}$ A wide dimensional scaling can be achieved in conversion to electrical energy using piezoelectric transducers. ${ }^{5,6}$

${ }^{*}$ Corresponding author. 
The classic approach is to tune the mechanical part of the harvester to resonate. This approach is good for stable, narrow band excitations but performs poorly in transient and changeable conditions of ambient excitation. ${ }^{1,4}$ Unfortunately, the small levels of energy available off-resonance is often insufficient and a tuning process has to be employed. Thus, the ideal device is a broadband harvester based on nonlinear characteristics which is not critically sensitive to the specific frequency or amplitude of excitation. ${ }^{6-8}$ The most promising approach is a nonlinear mechanical resonator with a dual well potential. ${ }^{9-11}$

More recently, Friswell et al. ${ }^{12}$ studied a piezoelastic system consisting of a cantilever beam with a tip mass that was mounted vertically and exposed to harmonic excitation in the horizontal direction at its base. For a fairly large tip mass, the vertical position destabilizes and the beam buckles giving a double well potential due to gravitational loading. The deterministic model was simulated and validated using an experimental device with three different tip masses, representing three interesting cases: a linear system a low natural frequency, nonbuckled beam and a buckled beam. The most practical configuration appeared to be the pre-buckled case, where the proposed system has a low natural frequency, a high level of harvested power, and an increased bandwidth over a linear harvester. The effects of random excitation and the combined harmonic and stochastic excitation components in horizontal directions were studied in two other papers. ${ }^{13,14}$

In the present paper, we follow the above mentioned model ${ }^{12-14}$ and complete the analysis by simulating an inverted beam excited by random noise in the vertical direction. Similar approaches to randomly excited broadband piezoelastic devices have been discussed by authors in Refs. 11, 15-23. Also note that the nonlinear system considered in this paper bends due to the gravitational field (Fig. 1), in contrast to the magnetic systems discussed in previous papers. ${ }^{11,15-23}$

\section{Equations of Motion}

An arbitrary point on the beam denoted by mass element $d m$ at distance $x$ from the oscillating base exciting stochastically by function $\chi(t)$ in vertical direction and the point mass $m_{t}$ were considered, which undergoes a flexible body deflection to a new position due to the base excitation. The mass element of the beam $d m$ undergoes a horizontal displacement $v_{d m}$ and a vertical displacement $\chi-u_{d m}$ and a rotation of $\varphi$ of the tip beam point (Fig. 1). Note that in our calculations, the thickness of the beam is small compared with the length, so that the effect of rotary inertia of the beam mass, $\varphi_{d m}$, was neglected.

The kinetic energy of the system is ${ }^{12,24,25}$

$$
\begin{aligned}
T= & \frac{1}{2} \rho A \int_{0}^{L}\left[\dot{v}_{d m}(x, t)^{2}+\left(\dot{\chi}(t)-\dot{u}_{d m}(x, t)\right)^{2}\right] \mathrm{d} x \\
& +\frac{1}{2} m_{t}\left[\dot{v}(t)^{2}+(\dot{\chi}(t)-\dot{u}(t))^{2}\right]+\frac{1}{2} I_{0} \dot{\varphi}^{2}
\end{aligned}
$$




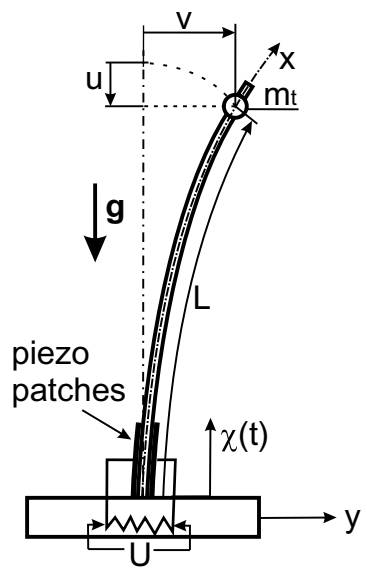

Fig. 1. The model of the inverted beam with a tip mass $m_{t}$ with its horizontal and vertical displacements $v$ and $u$. $L$ is the length of beam, while $x$ is the axis oriented along the beam. $\mathrm{g}$ denotes the gravitational acceleration vector while $\chi(t)$ denotes the kinematic vertical excitation.

and the potential energy consists of the elastic beam part and the gravity term of the beam with the tip mass, is

$$
\Pi=\frac{1}{2} E I \int_{0}^{L} \kappa(x, t)^{2} d x+g \rho A \int_{0}^{L}\left(-u_{d m}(x, t)\right) d x+m_{t} g(-u(t)) .
$$

A single degree of freedom model of the deflection of the beam is considered, where

$$
v_{d m}(x, t)=v_{d m}(L, t) \psi(x)=v(t) \psi(x),
$$

where displacement $\psi(x)$ is assumed to $\mathrm{be}^{25}$ :

$$
\psi(x)=1-\cos \left(\frac{\pi x}{2 L}\right)
$$

After some algebraic manipulations (see Ref. 12) we get the kinetic and potential energies in terms of $v$ :

$$
\begin{aligned}
T= & \frac{1}{2} \rho A \int_{0}^{L}\left[\dot{v}(t)^{2} \psi(x)^{2}+\left(\dot{\chi}(t)-v(t) \dot{v}(t) \int_{0}^{x} \psi^{\prime}(x)^{2} d x\right)^{2}\right] d x \\
& +\frac{1}{2} m_{t}\left[\dot{v}(t)^{2}+\left(\dot{\chi}(t)-v(t) \dot{v}(t) \int_{0}^{L_{t}} \psi^{\prime}(x)^{2} d x\right)^{2}\right] \\
& +\frac{1}{2} I_{0} \dot{v}(t)^{2} \psi^{\prime}\left(L_{t}\right)^{2}\left(1+\frac{1}{2} v(t)^{2} \psi^{\prime}\left(L_{t}\right)^{2}\right)^{2}
\end{aligned}
$$


and

$$
\begin{aligned}
\Pi= & \frac{1}{2} E I \int_{0}^{L}\left(v(t) \psi^{\prime \prime}(x)+\frac{1}{2} v(t)^{3} \psi^{\prime}(x)^{2} \psi^{\prime \prime}(x)\right)^{2} d x \\
& -\frac{1}{2} \rho A g v(t)^{2} \int_{0}^{L}\left(\int_{0}^{x} \psi^{\prime}(x)^{2} d x\right) d x-\frac{1}{2} m_{t} g v(t)^{2} \int_{0}^{L_{t}} \psi^{\prime}(x)^{2} d x,
\end{aligned}
$$

where the prime denotes differentiation with respect to $x$.

Taking $\psi$ as a displacement of the beam expressed by Eq. (2.4) and denoting the constants that occur in Eqs. (2.5) and (2.6) by $N_{1}-N_{8}$, the final kinetic and potential energies are given by:

$$
\begin{aligned}
T= & \frac{1}{2} \rho A\left[\dot{v}(t)^{2} N_{1}+\dot{\chi}(t)^{2} L-2 \dot{\chi}(t) v(t) \dot{v}(t) N_{2}+v(t)^{2} \dot{v}(t)^{2} N_{3}\right] \\
& +\frac{1}{2} m_{t}\left[\dot{v}(t)^{2}+\dot{\chi}(t)^{2}-2 \dot{\chi}(t) v(t) \dot{v}(t) N_{4}+v(t)^{2} \dot{v}(t)^{2} N_{4}^{2}\right] \\
& +\frac{1}{2} I_{0} \dot{v}(t)^{2} N_{5}^{2}\left(1+\frac{1}{2} v(t)^{2} N_{5}^{2}\right)^{2}
\end{aligned}
$$

and

$$
\Pi=\frac{1}{2} E I\left(v(t)^{2} N_{6}+v(t)^{4} N_{7}+\frac{1}{4} v(t)^{6} N_{8}\right)-\frac{1}{2} g v(t)^{2}\left(\rho A N_{2}+m_{t} N_{4}\right),
$$

where constants $N_{1}-N_{8}$ are:

$$
\begin{aligned}
& N_{1}=\int_{0}^{L} \psi(x)^{2} d x=L\left(\frac{3}{2}-\frac{4}{\pi}\right), \quad N_{2}=\int_{0}^{L} \int_{0}^{x} \psi^{\prime}(\xi)^{2} d \xi d x=\frac{1}{16}\left(\pi^{2}-4\right), \\
& N_{3}=\int_{0}^{L}\left(\int_{0}^{x} \psi^{\prime}(\xi)^{2} d \xi\right)^{2} d x=\frac{\pi^{2}}{384 L}\left(2 \pi^{2}-9\right), \quad N_{4}=\int_{0}^{L_{t}} \psi^{\prime}(x)^{2} d x=\frac{\pi^{2}}{8 L_{t}} \\
& N_{5}=\psi^{\prime}\left(L_{t}\right)=\frac{\pi}{2 L_{t}}, \quad N_{6}=\int_{0}^{L} \psi^{\prime \prime}(x)^{2} d x=\frac{\pi^{4}}{32 L^{3}}, \\
& N_{7}=\int_{0}^{L} \psi^{\prime \prime}(x)^{2} \psi^{\prime}(x)^{2} d x=\frac{\pi^{6}}{512 L^{5}}, \quad N_{8}=\int_{0}^{L} \psi^{\prime \prime}(x)^{2} \psi^{\prime}(x)^{4} d x=\frac{\pi^{8}}{4096 L^{7}} .
\end{aligned}
$$

Lagrange's method is applied to derive the equation of motion of the beam-mass system, given by

$$
\frac{d}{d t}\left(\frac{\partial \mathbb{L}}{\partial \dot{v}}\right)-\frac{\partial \mathbb{L}}{\partial v}=0
$$


where $\mathbb{L}=T-\Pi$ is the Lagrangian. Neglecting the terms higher than third order, the equation of motion becomes

$$
\begin{aligned}
{\left[m_{t}+\right.} & \left.\rho A N_{1}+I_{0} N_{5}^{2}+\left(\rho A N_{3}+m_{t} N_{4}^{2}+I_{0} N_{5}^{4}\right) v^{2}\right] \ddot{v} \\
& +\left[\rho A N_{3}+m_{t} N_{4}^{2}+I_{0} N_{5}^{4}\right] v \dot{v}^{2} \\
& +\left[E I\left(N_{6}+2 N_{7} v^{2}\right)-(g+\ddot{\chi})\left(m_{t} N_{4}+\rho A N_{2}\right)\right] v=0
\end{aligned}
$$

where $\ddot{\chi}$ is the acceleration of the base of the beam and is assumed to be stochastic and of the form

$$
\ddot{\chi}=\Gamma(t),
$$

where $\Gamma(t)$ denotes the stochastic factor. The other symbols used above are defined in Tables 1 and 2 .

The equilibrium position with no forcing is obtained by setting the acceleration and velocity to zero in Eq. (2.11), to give:

$$
\left[E I\left(N_{6}+2 N_{7} v^{2}\right)-g\left(m_{t} N_{4}+\rho A N_{2}\right)\right] v=0 .
$$

The above equation has one solution $v=0$ or three solutions depending on whether the following inequality is satisfied:

$$
g\left(m_{t} N_{4}+\rho A N_{2}\right)>E I N_{6} .
$$

Table 1. System parameters.

\begin{tabular}{ll}
\hline Symbol and value & \multicolumn{1}{c}{ Description } \\
\hline$L=0.3 \mathrm{~m}$ & Length of the beam \\
$m_{t}=0.02 \mathrm{~kg}$ & The tip mass \\
$m_{b}=0.0096 \mathrm{~kg}$ & The beam mass \\
$\rho=7850 \mathrm{~kg} / \mathrm{m}^{3}$ & Density of the beam mass \\
$A=4.064 \mathrm{~mm}^{2}$ & Area of transverse beam \\
$E=210 \mathrm{GPa}$ & Young modulus \\
$I=2.18 \times 10^{-14} \mathrm{~m}^{4}$ & Geometrical moment of inertia \\
$I_{0}=0.0021 \mathrm{~kg} \mathrm{~m} \mathrm{~m}^{2}$ & Mass moment of inertia for the tip mass \\
$\chi$ & Kinematic displacement of the base \\
$C_{p}=38.9 \mathrm{nF}$ & Capacitance of the piezoelectric patches \\
$R=50 \mathrm{k} \Omega$ & Load resistance \\
$L_{p}=28 \mathrm{~mm}$ & Active length of piezoelectric layers \\
$b_{b}=16 \mathrm{~mm}$ & Beam width \\
$h_{b}=2.54 \mathrm{~mm}$ & Beam thickness \\
$h_{p}=300 \mu \mathrm{m}$ & Piezoelectric layers thickness \\
$e_{31}=-5.157 \mathrm{C} / \mathrm{m}^{2}$ & Piezoelectric constant \\
$c=0.05 \mathrm{kq} \mathrm{m}$ & Effective damping coefficient \\
\hline
\end{tabular}

Table 2. Effective parameters in SI units (see Eqs. (2.11)-(3.1)).

\begin{tabular}{cccccccc}
\hline$N_{1}[m]$ & $N_{2}[-]$ & $N_{3}\left[m^{-1}\right]$ & $N_{4}\left[m^{-1}\right]$ & $N_{5}\left[m^{-1}\right]$ & $N_{6}\left[m^{-3}\right]$ & $N_{7}\left[m^{-5}\right]$ & $N_{8}\left[m^{-7}\right]$ \\
\hline 0.0680281 & 0.36685 & 0.920067 & 4.11234 & 5.23599 & 112.742 & 772.722 & 10592.3 \\
\hline
\end{tabular}


Then the nonzero stable equilibrium points are:

$$
v_{0}= \pm \sqrt{\frac{g\left(m_{t} N_{4}+\rho A N_{2}\right)-I E N_{6}}{2 E I N_{7}}}
$$

The linearized equation of motion for the free response about the equilibrium solution at $v=0$ is:

$$
\left[m_{t}+\rho A N_{1}+I_{0} N_{5}^{2}\right] \ddot{v}+\left[E I N_{6}-g\left(m_{t} N_{4}+\rho A N_{2}\right)\right] v=0 .
$$

For $v=0$, the natural frequency for small vibrations is given by:

$$
\omega_{0}^{2}=\frac{E I N_{6}-g\left(m_{t} N_{4}+\rho A N_{2}\right)}{m_{t}+\rho A N_{1}+I_{0} N_{5}^{2}} .
$$

About the second equilibrium, given by the buckled position $v=v_{0 b}$, the linearized equation of motion for free response is:

$$
\left[m_{t}+\rho A N_{1}+I_{0} N_{5}^{2}+\left(\rho A N_{3}+m_{t} N_{4}^{2}+I_{0} N_{5}^{4}\right) v_{0 b}^{2}\right] \ddot{\eta}+4 E I N_{7} v_{0 b}^{2} \eta=0,
$$

where $v=v_{0 b}+\eta$, and the natural frequency about both buckled equilibrium positions are:

$$
\omega_{0 b}^{2}=\frac{4 E I N_{7} v_{o b}^{2}}{m_{t}+\rho A N_{1}+I_{0} N_{5}^{2}+\left(\rho A N_{3}+m_{t} N_{4}^{2}+I_{0} N_{5}^{4}\right) v_{0 b}^{2}} .
$$

Figure 2 shows the influence of the tip mass $m_{t}$ on the natural frequencies and the equilibrium positions. In Fig. $2(\mathrm{a})$ one can see the interesting $\omega_{0 b}=0$ limit for $m_{t} \approx 10 \mathrm{~g}$. This point coincides with the bifurcation of the equilibrium positions and is characterized by the increasing flatness of the potential.

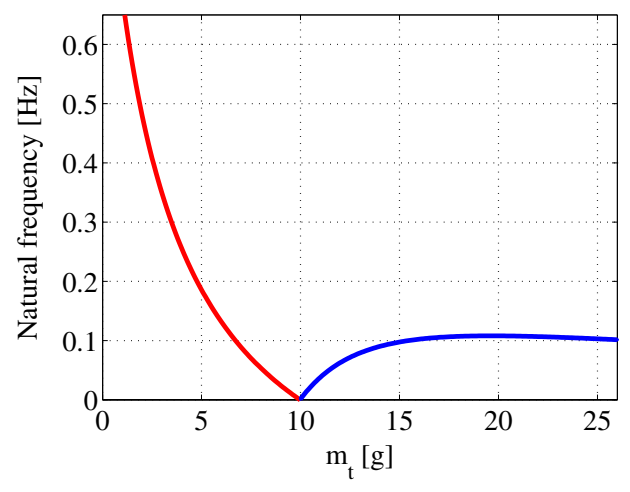

(a)

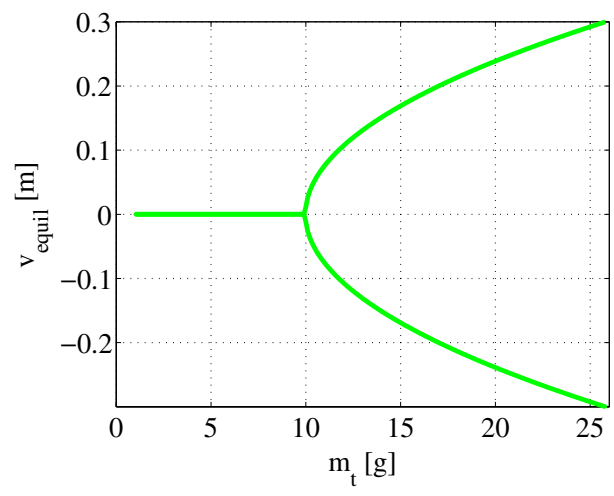

(b)

Fig. 2. The natural frequencies versus tip mass $m_{t}$ (a), and the equilibrium positions versus tip mass $m_{t}$ (b). 


\section{The Coupled Piezoelectric Model}

Using the derivation of the coupled electro-mechanical model provided by authors, ${ }^{12}$ the equation of motion combined with the electrical equation becomes

$$
\begin{aligned}
{\left[m_{t}+\right.} & \left.\rho A N_{1}+I_{0} N_{5}^{2}+\left(\rho A N_{3}+m_{t} N_{4}^{2}+I_{0} N_{5}^{4}\right) v^{2}\right] \ddot{v} \\
& +\left[\rho A N_{3}+m_{t} N_{4}^{2}+I_{0} N_{5}^{4}\right] v \dot{v}^{2} \\
& +\left[E I\left(N_{6}+2 N_{7} v^{2}\right)-(g+\ddot{\chi})\left(m_{t} N_{4}+\rho A N_{2}\right)\right] v+c \dot{v}-D U=0, \\
C_{p} \dot{U} & +\frac{U}{R}+D \dot{v}=0,
\end{aligned}
$$

where $c$ is an effective damping coefficient and $U$ is the voltage across the load resistor connected to the piezoelectric patch. $C_{p}$ is the capacitance of the piezoelectric patches and $R$ is the load resistance. Two piezoelectric patches are attached to the beam and connected in parallel. The constant for the electromechanical coupling is given by

$$
D=e_{31} b_{p}\left(h_{p}+h_{b}\right) \int_{0}^{L_{p}} \psi^{\prime \prime}(x) \mathrm{d} x=e_{31} b_{p}\left(h_{p}+h_{b}\right) \psi^{\prime}\left(L_{p}\right),
$$

where $h_{b}$ is the beam thickness, $h_{p}$ is the piezoelectric layer thickness, $b_{p}$ is the piezoelectric layer width, $L_{p}$ denotes active length of piezoelectric layer, and $e_{31}$ is the piezoelectric constant.

\section{Results of Numerical Simulations}

Based on Eq. (3.1), we performed the simulations on the physical system (Fig. 1) for the given system parameters (Table 1). To model the random force, we adopted the

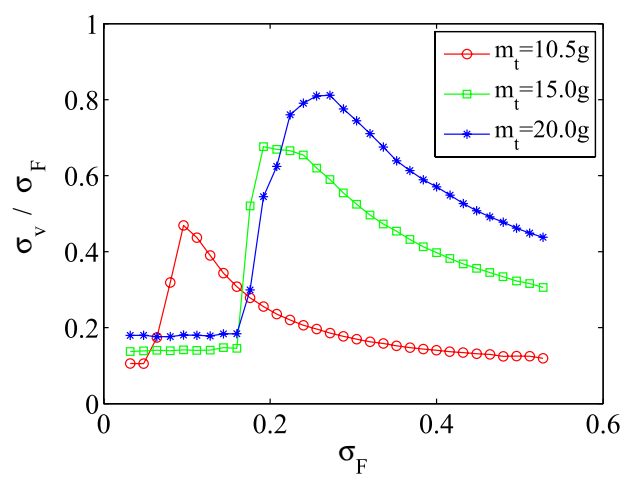

(a)

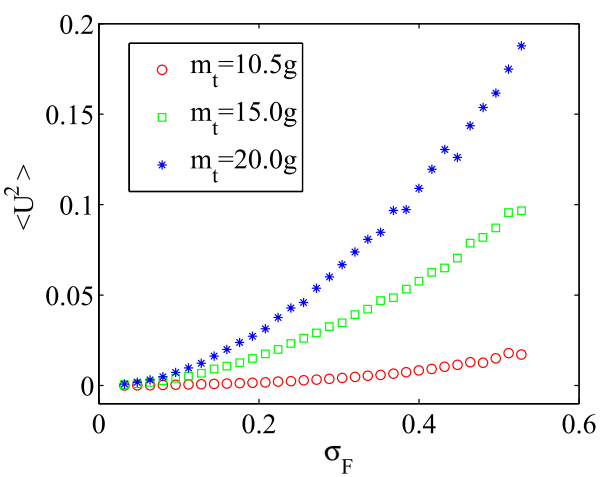

(b)

Fig. 3. The displacement signal to noise ratio $\sigma_{v} / \sigma_{F}$ versus $\sigma_{F}$ for tip mass $m_{t}=10.5,15.0$ and $20.0 \mathrm{~g}$ (a), the mean square of the generated voltage $\left\langle U^{2}\right\rangle$ for the corresponding tip mass $m_{t}$ (b), the number of hops (c) and the mean displacement (d). The initial conditions for each noise level were $[v, \dot{v}, U]_{t=0}=[0.2021 \mathrm{~m}$, $0,0]$. Each point on the figures is an average of 10 different noise realizations. 


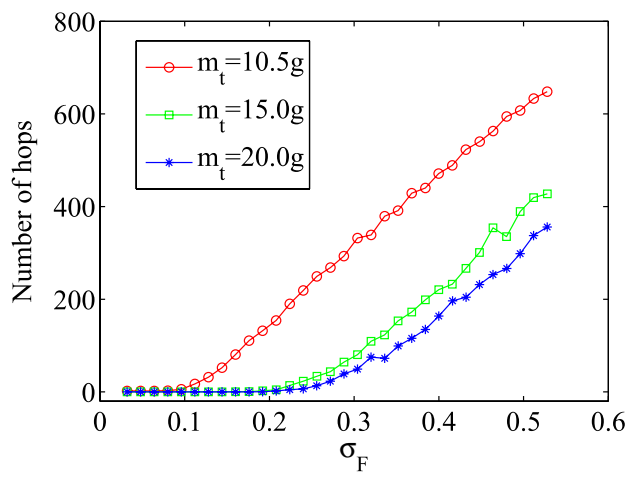

(c)

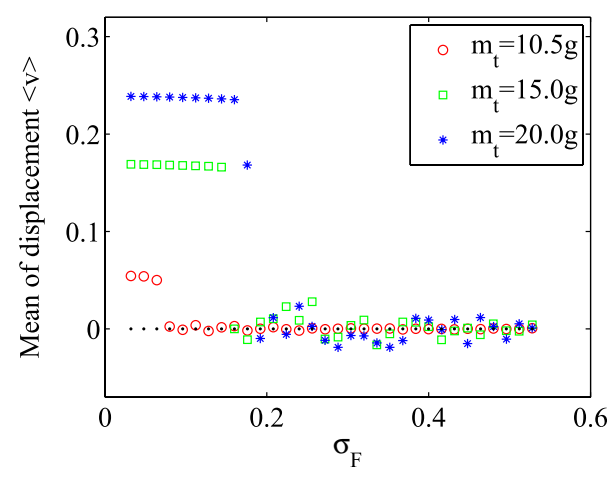

(d)

Fig. 3. (Continued)

Runge-Kutta-Murayama algorithm ${ }^{19,26}$ and used white noise of Gaussian distribution. The main results are summarized in Fig. 3. The simulation time period was fixed as $t \in[0,10800 \mathrm{~s}]$, while the time step was $\delta t=0.01 \mathrm{~s}$. The initial time intervals of each simulation $[0,7200 \mathrm{~s}]$ were neglected as transients in the statistical estimation of all of the presented parameters. The initial conditions for each noise level were $[v, \dot{v}, U]_{t=0}=[0.2021 \mathrm{~m}, 0,0]$. Each point on the figures is an average of 10 different noise realizations.

Figure 3(a) shows the displacement signal to noise ratio in terms of the corresponding standard deviations $\sigma_{v}$ and $\sigma_{F}, \sigma_{v} / \sigma_{F}$ versus $\sigma_{F}$, for tip mass $m_{t}=10.5$, 15.0 and $20.0 \mathrm{~g}$, respectively. Here, one can see visible peak regions. This is a signature the stochastic resonance. ${ }^{27} \mathrm{~A}$ similar phenomenon (stochastic coherence

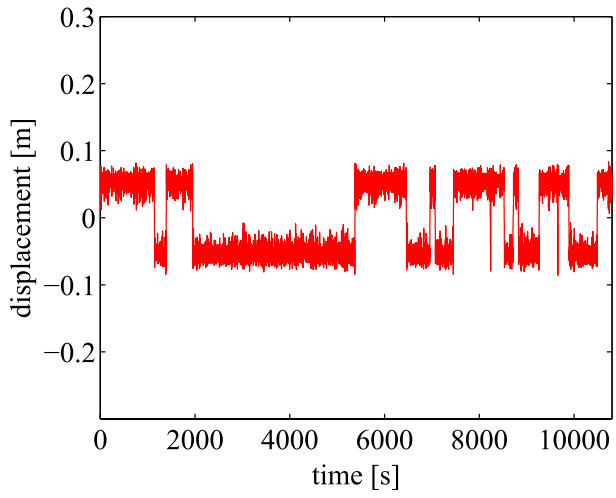

(a)

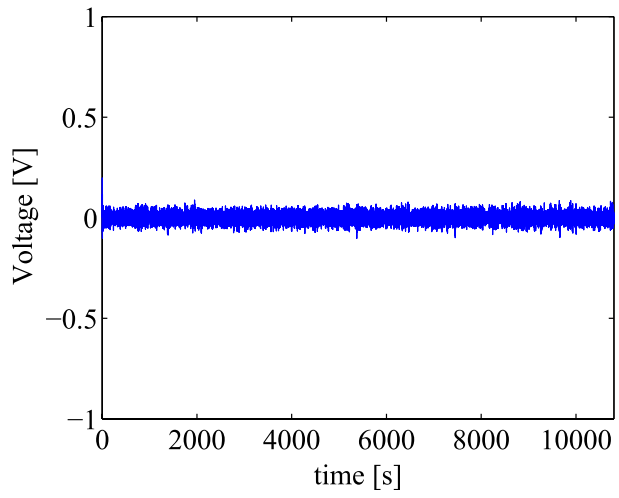

(b)

Fig. 4. Time series of displacement (a) and voltage (b). The phase portrait (c) at noise level $\sigma_{F}=0.1$ and tip mass $m_{t}=10.5 \mathrm{~g}$. The number of jumps between the potential well (d) against simulation time at noise level $\sigma_{F}=0.1$ by tip masses $m_{t}=10.5 \mathrm{~g}$ (black) and $m_{t}=15 \mathrm{~g}$ (red), respectively. 
Energy Harvesting in a Nonlinear Cantilever Piezoelastic Beam System

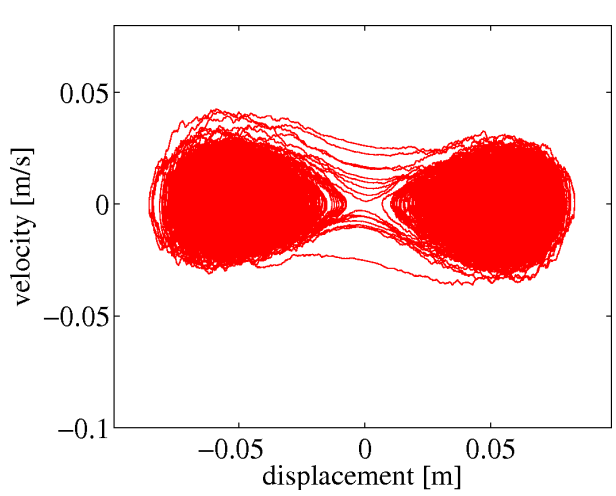

(c)

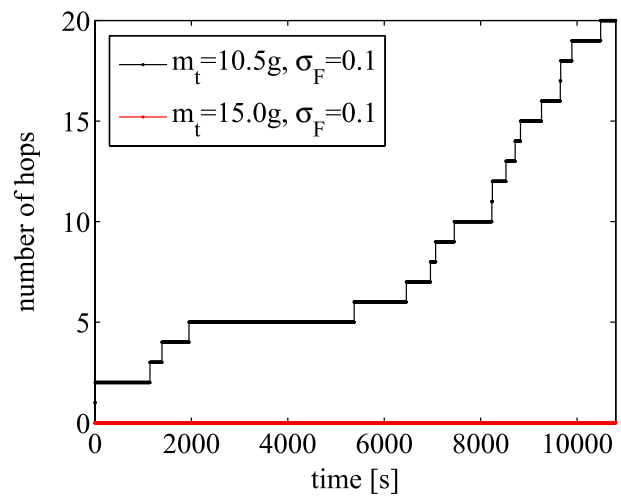

(d)

Fig. 4. (Continued)

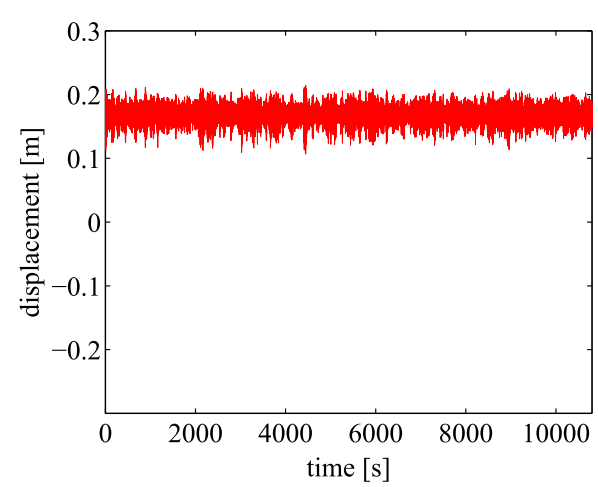

(a)

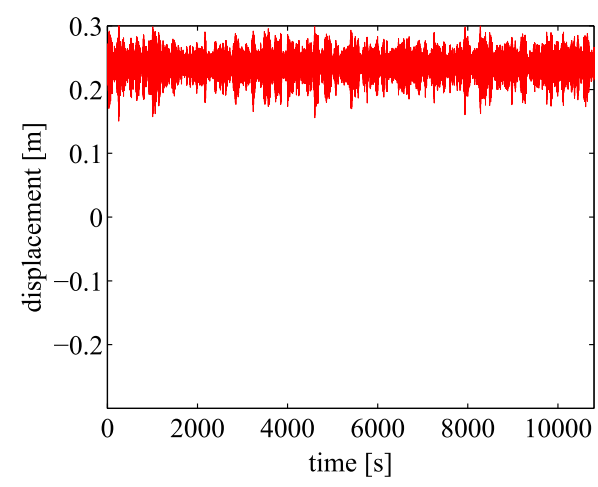

(c)

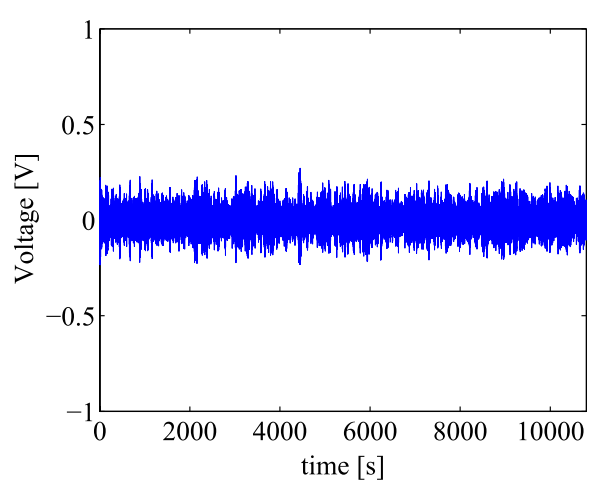

(b)

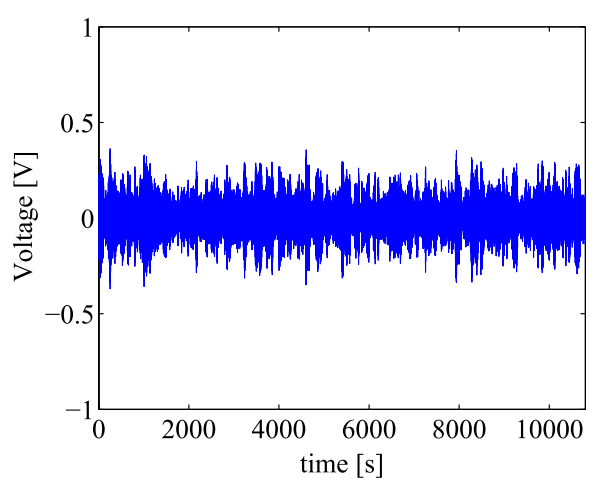

(d)

Fig. 5. Time series of displacements (a) and voltages (b) at constant noise $\sigma_{F}=0.1$ and increased tip masses $m_{t}=15 \mathrm{~g}(\mathrm{a}, \mathrm{b})$ and $m_{t}=20 \mathrm{~g}(\mathrm{c}, \mathrm{d})$. 
resonance) has been reported in the systems with purely random excitation. ${ }^{11,15,28}$ Generally, this phenomenon is caused when the potential barrier is overcome. Note that the case $m_{t}=10.5 \mathrm{~g}$ is characterized by an almost immediate pass through the barrier (see Fig. 2(b)).

Simultaneously calculated, the mean square of the generated voltage $\left\langle U^{2}\right\rangle$ for the corresponding tip mass $m_{t}$ (Fig. $3(\mathrm{~b})$ ) does not indicate any spectacular increase in the power output when the potential barrier is overcome. The results show a continuous increase in the power output with increasing noise level as a square polynomial function of $\sigma_{F}$. Note also that the larger mass $m_{t}$ causes a higher power output.

The number of hops between the potential wells (Fig. 3(c)) and the average displacement (Fig. 3(d)) illustrate additional features of the dynamics of the vertical cantilever resonator.

Finally, we show examples of the displacement and voltage time series, as well as the corresponding phase portraits, in the case of particular stochastic simulations with $m_{t}=10.5,15.0$ and $20.0 \mathrm{~g}$, for a low noise level $\sigma_{F}=0.1$ (Figs. 4 and 5 ) and a higher noise level $\sigma_{F}=0.3$ (Fig. 6). While the intermittent stochastic response is present for the case with the tip mass $m_{t}=10.5 \mathrm{~g}$ (see the Figs. 4 (a) and $4(\mathrm{c})$ ), the larger $m_{t}$ leads to single well dynamics (see Figs. 5(a)-5(d)). Note that in spite of intermittent displacement (Figs. 4(a) and 6(a)) motion, we observe a symmetry in the voltage (Figs. 4(b) and 6(c)). This is due to the coupling of mechanical into electrical parts of the model equations of motion (see Eq. (2.3)) which is realized through velocity, $\dot{v}$. The cross-barrier vibration evolve with changing system parameters. Fairly rare jumps between the potential wells are visible in Fig. 4(a) while more frequent jumps are reported in Fig. 6(a).

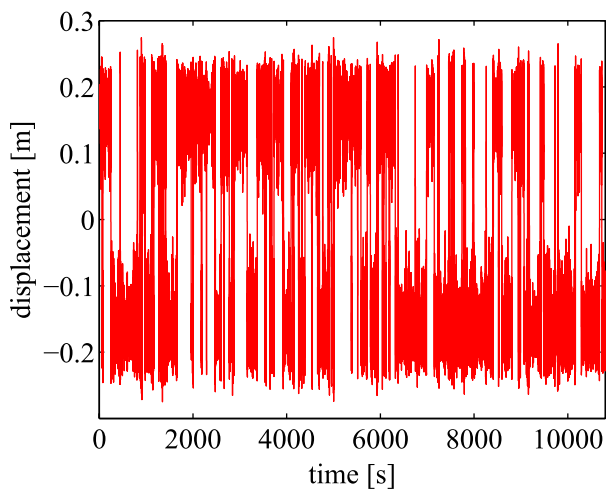

(a)

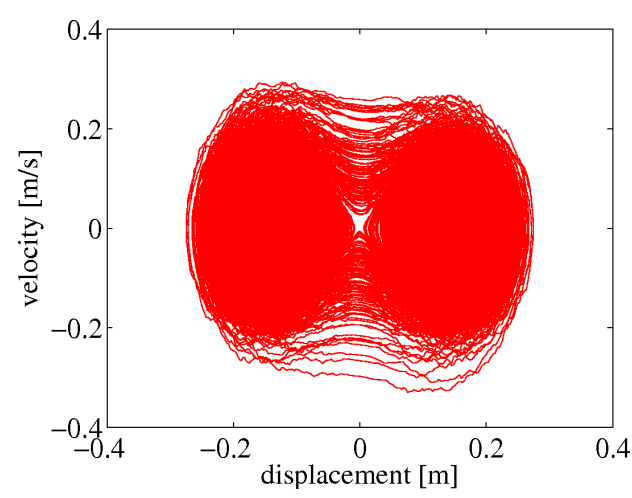

(b)

Fig. 6. Time series of displacements (a), phase portrait (b), voltage (c) and jumps (d) at noise level $\sigma_{F}=0.3$ and tip mass $m_{t}=15 \mathrm{~g}$. 


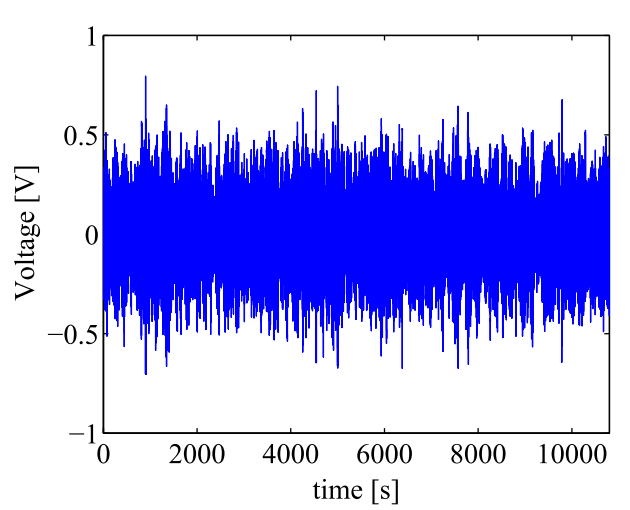

(c)

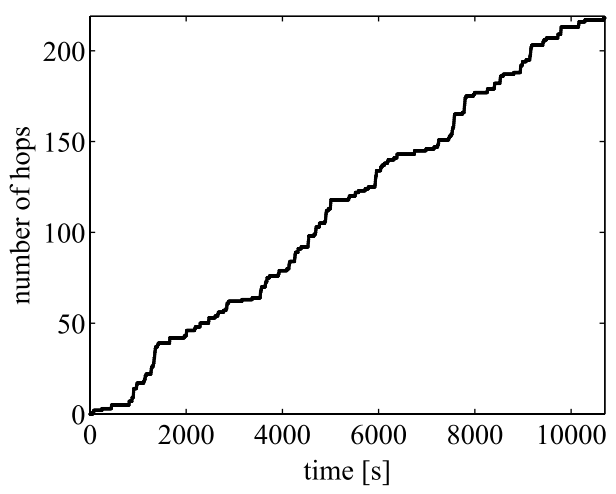

(d)

Fig. 6. (Continued)

\section{Conclusion}

We studied the response of a vertical cantilever beam under stochastic vertical excitation. Our results show interesting transitions from single to double well vibrations. However the final conclusion shows that higher efficiency in displacement vibrations (stochastic coherence resonance) has a fairly small influence on the power output. Note that this result is valid for random force excitation, without any periodic component where the hopping introduces an additional time scale. However, in the presence of an harmonic excitation part the situation could be different, as studied in the similar system with a horizontal excitation. ${ }^{14}$ In comparison to the horizontal excitation, the effect of small amplitude excitation of the pre-buckled beam of the present case is not effective as the vertical inertial force is not linearly coupled to small oscillations in the horizontal direction. The situation is changing with increasing tip mass crossing buckling conditions. To draw more conclusions, more detailed comparative studies including experiments between these two cases with different excitation directions should be performed.

\section{Acknowledgments}

$\mathrm{MB}$ and GL were partially supported by the Polish National Science Center under the Grant No. 2012/05/B/ST8/00080.

\section{References}

1. C. B. Williams and R. B. Yates, Analysis of a micro-electric generator for microsystems, Sensors Actuators A 52 (1996) 8-11.

2. S. Roundy, P. Wright and J. Rabaey, Energy Scavenging for Wireless Sensor Networks (Kluwer Academics Boston, MA, 2003). 
3. S. R. Anton, A. Erturk and D. J. Inmann, Multifunctional self-charging structures using piesoceramics and thin-film batteries, Smart Mater. Struct. 19 (2010) 115021.

4. H. S. Kim, J.-H. Kim and J. Kim, A review of piezoelectric energy harvesting based on vibrations, Int. J. Precision Eng. Manufact. 12 (2011) 1129-1141.

5. P. D. Mitcheson, E. M. Yeatman, G. K. Rao, A. S. Holmes and T. C. Green, Energy harvesting from human and machine motion for wireless electronic devices, Proc. IEEE 96 (2008) 1457-1486.

6. L. Tang, Y. Yang and C. K. Soh, Toward broadband vibration-based energy harvesting, J. Intell. Mater. Syst. Struct. 21 (2010) 1867-1897.

7. A. Erturk and D. Inman, Piezoelectric Energy Harvesting (John Wiley \& Sons Ltd., Chichester, UK, 2011).

8. S. Priya, Advances in energy harvesting using low profile piezoelectric transducers, J. Electroceram. 19 (2007) 167-184.

9. A. Erturk, J. Hoffmann and D. J. Inman, A piezomagnetoelastic structure for broadband vibration energy harvesting, Appl. Phys. Lett. 94 (2009) 254102.

10. E. S. Leland and P. K. Wright, Resonance tuning of piezoelectric vibrations energy scavenging generators using compressive axial preload, Smart Mater. Struct. 15 (2006) 1413-1420.

11. G. Litak, M. I. Friswell and S. Adhikari, Magnetopiezoelastic energy harvesting driven by random excitations, Appl. Phys. Lett. 96 (2010) 214103.

12. M. I. Friswell, S. F. Ali, S. Adhikari, A. W. Lees, O. Bilgen and G. Litak, Nonlinear piezoelectric vibration energy harvesting from a vertical cantilever beam with tip mass, J. Int. Mater. Syst. Struct. 23 (2013) 1505-1521.

13. M. Borowiec, G. Litak, M. I. Friswell, S. F. Ali, S. Adhikari, A. W. Lees and O. Bilgen, Energy harvesting in piezoelastic systems driven by random excitations, Int. J. Struct. Stab. Dynam. 13 (2013) 1340006.

14. M. I. Friswell, O. Bilgen, S. F. Ali, G. Litak and S. Adhikari, The effect of noise on the response of a vertical cantilever beam energy harvester, Zeit. Angew. Math. Mech., doi: 10.1002/zamm.201300183 (2014).

15. F. Cottone, H. Vocca and L. Gammaitoni, Nonlinear energy harvesting, Phys. Rev. Lett. $102(2009) 80601$.

16. E. Halvorsen, Energy harvesters driven by broadband random vibrations, J. Micromech. Syst. 17 (2008) 1061-1071.

17. L.-C. J. Blystad and E. Halvorsen, An energy harvester driven by colored noise, Smart Mater. Struct. 20 (2011) 025011.

18. M. Ferrari, M. Bau, M. Guizzetti and V. Ferrari, A single-magnet nonlinear piezoelectric converter for enhanced energy harvesting from random vibrations, Sensor Actuators A Phys. 172 (2011) 287-292.

19. G. Litak, M. Borowiec, M. I. Friswell and S. Adhikari, Energy harvesting in a magnetopiezoelastic system driven by random excitation with uniform and Gaussian distributions, J. Theor. Appl. Mech. 49 (2011) 757-764.

20. N. A. Khovanova and I. A. Khovanov, The role of excitations statistic and nonlinearity in energy harvesting from random impulsive excitations, App. Phys. Lett. 99 (2011) 144101.

21. M. F. Daqaq, Transduction of a bistable inductive generator driven by white and exponentially correlated Gaussian noise, J. Sound Vibr. 330 (2011) 2554-2564.

22. W. Martens, U. von Wagner and G. Litak, Stationary response of nonlinear magnetopiezoelectric energy harvester systems under stochastic excitation, Eur. Phys. J. Special Topics 222 (2013) 1665-1673.

23. P. Kumar, S. Narayanan, S. Adhikari and M. I. Friswell, FokkerPlanck equation analysis of randomly excited nonlinear energy harvester, J. Sound Vibr. 333 (2014) 2040-2053. 
24. L. D. Zavodney and A. H. Nayfeh, The nonlinear response of a slender bean carrying lumped mass to a principal parametric excitation: Theory and experiment, Int. J. Nonlin. Mech. 24 (1989) 105-125.

25. E. Esmailzadeh and G. Nakhaie-Jazar, Periodic behavior of a cantilever beam with end mass subjected to harmonic base excitation, Int. J. Nonlin. Mech. 33 (1998) 567-577.

26. A. Naess and V. Moe, Efficient path integration methods for nonlinear dynamic systems, Probab. Eng. Mech. 15 (2000) 221-231.

27. L. Gammaitoni, P. Hanggi, P. Jung and F. Marchesoni, Stochastic resonance, Rev. Mod. Phys. 70 (1998) 223-287.

28. C. R. McInnes, D. G. Gorman and M. P. Cartmell, Enhanced vibrational energy harvesting using nonlinear stochastic resonance, J. Sound Vibr. 318 (2008) 655-662. 